\title{
ON-OFF INTERMITTENCY AND PECULIAR PROPERTIES OF ATTRACTORS IN A SIMPLE MODEL OF CHAOS IN FERROMAGNETIC RESONANCE
}

\author{
A. Krawiecki and A. SUkiennicki \\ Institute of Physics, Warsaw University of Technology \\ Koszykowa 75, 00-662 Warszawa, Poland
}

(Received March 31, 1995)

\begin{abstract}
Some non-trivial phenomena in chaos in ferromagnetic resonance above the first-order Suhl instability threshold are obtained numerically from a simple model of three interacting modes. They include sudden changes of the correlation dimension of the attractor and the largest Lyapunov exponent with the rise of the rf field amplitude. Numerical evidence is provided that these effects may result from the on-off intermittency in the system of interacting modes. These results are qualitatively similar to the experimental ones, known from the literature, obtained for in-plane magnetized thin films.
\end{abstract}

PACS numbers: 05.45.+b, 76.50.+g, 75.30.Ds

\section{Introduction}

In the last years, high-power ferromagnetic resonance experiments provided an useful tool in the study of nonlinear dynamics. Auto-oscillations of the magnetization and chaos have been observed experimentally and analyzed theoretically for various resonance configurations and sample shapes (see e.g. [1-9]).

In this paper we present numerical results, obtained for a very simple model of low-dimensional chaos above the first-order Suhl instability threshold. This instability occurs in perpendicular resonance, when a mode driven by the transverse rf field (e.g. the uniform one) decays into a pair of parametric modes with half the pumping frequency and opposite wave vectors (e.g. spin waves) [10]. The minimum rf field amplitude necessary for the occurrence of the instability is called the instability threshold. Usually, only one parametric mode pair goes unstable exactly at the threshold and its amplitude grows exponentially in time, but it was verified experimentally that other mode pairs may also be excited as the rf field amplitude rises [11]. Nonlinear interactions among the modes lead to the appearance of 
the auto-oscillations and chaos in the time dependence of the magnetization. The measured quantity is usually the rf field energy absorption, which is proportional to the driven mode amplitude, and the rf field amplitude plays a role of the control parameter.

In this paper we show that such quantities as the correlation dimension of the attractor or the largest Lyapunov exponent, commonly utilized to characterize the underlying chaotic dynamics, may undergo sudden changes with the rise of the rf field amplitude due to the changes of the number of modes, which are excited via the decay of the driven mode. We also argue that the transition between chaotic states with different number of excited modes may result from the on-off intermittency, recently described in Refs. $[12,13]$. Our numerical results are then compared to the experimental ones, obtained by other authors, concerning in-plane magnetized films [7], and to the results of the numerical modelling of chaos in parallel pumping [8] with the use of the stroboscopic model [14].

\section{The model}

Our discussion concerns the case of resonant excitation of the driven mode by the rf field (coincidence regime). Following a simple approach to the first-order Suhl instability problem [10] we consider only interactions between the rf field and the driven mode as well as three-mode interactions between the driven mode and parametric mode pairs, conserving energy. The mode driven by the rf field will be denoted by zero, and the parametric mode pairs by $k,-k ; k=1,2, \ldots$ Equations of motion for the complex mode amplitudes $c_{0}, c_{k}, c_{-k}$ may be obtained either from the Landau-Lifshitz equation (see e.g. $[10,4]$ ) or from the Hamiltonian $\mathcal{H}$ written in terms of the mode creation and annihilation operators (see e.g. [3])

$$
\begin{aligned}
& \mathcal{H}=h_{T} \cos \omega t\left(I_{0} c_{0}^{*}+\text { c.c. }\right)+\sum_{k} \omega_{k} c_{k}^{*} c_{k}+\sum_{k} V_{0, k} c_{0}^{*} c_{k} c_{-k}+\text { c.c. } \\
& \frac{\partial c_{k}}{\partial t}=\mathrm{i} \frac{\partial \mathcal{H}}{\partial c_{k}^{*}}, \quad \frac{\partial c_{k}^{*}}{\partial t}=-\mathrm{i} \frac{\partial \mathcal{H}}{\partial c_{k}} .
\end{aligned}
$$

Here, $h_{T}$ is the $\mathrm{rf}$ field amplitude with frequency $\omega, I_{0}$ is the coupling coefficient between the $r f$ field and the driven mode, $V_{0, k}$ are the coupling coefficients between the driven mode and parametric mode pairs and the star denotes complex conjugate. Taking into account that the amplitudes of the parametric modes, belonging to one pair $k$, differ only by a constant phase factor $q_{k}$ [15], i.e. $c_{-k}=c_{k} \exp \left(\mathrm{i} q_{k}\right)$, these equations are rewritten in new variables $C_{k}=c_{k} \exp \left(\mathrm{i} q_{k} / 2\right)$. Then the rapidly varying part from the time dependence of these variables is extracted by decomposing them into a product $C_{0}(t)=u_{0}(t) \exp (-\mathrm{i} \omega t), C_{k}(t)=u_{k}(t) \exp (-\mathrm{i} \omega t / 2)$, where $u_{0, k}$ vary slowly in time in comparison with the $\mathrm{rf}$ field frequency. On the right-hand side of the equations of motion terms with slow time dependence are only retained. The final form of the equations of motion is as follows (see e.g. [4])

$$
\begin{aligned}
& \dot{u}_{0}=-\mathrm{i} I_{0} \frac{h_{T}}{2}-\left(\eta_{0}+\mathrm{i} \Delta \omega_{0}\right) u_{0}-\mathrm{i} \sum_{k} V_{0, k} u_{k}^{2}, \\
& \dot{u}_{k}=-\left(\eta_{k}+\mathrm{i} \Delta \omega_{k}\right) u_{k}-\mathrm{i} V_{0, k}^{*} u_{k}^{*} u_{0} .
\end{aligned}
$$


Here, $\eta_{0, k}$ denotes phenomenological damping and $\Delta \omega_{0}=\omega_{0}-\omega, \Delta \omega_{k}=\omega_{k}-\omega / 2$ - the average detuning of the two modes of the pair from $\omega / 2$. The coupling $V_{0, k}$ depends in general on the type, wave vector and propagation direction of the parametric modes. If the boundary conditions at the sample surface are accounted for, one should use instead of spin-waves magnetic eigenmodes of the sample as the parametric modes, e.g. magnetostatic modes or dipole-exchange spin-waves $[4,16]$. From Eqs. (3), using standard methods [10], one obtains the threshold field for the $k$-th mode instability

$$
h_{T \text { crit }}=\frac{2\left|\delta_{0}\right|\left|\delta_{k}\right|}{\left|I_{0}\right|\left|V_{0, k}\right|}, \quad \delta_{0, k}=\eta_{0, k}+\mathrm{i} \Delta \omega_{0, k} .
$$

Above this threshold, amplitude of the $k$-th mode may grow exponentially in time, draining energy from the driven mode. Equation (4) is then minimized with respect to the wave vectors of the parametric modes to yield the Suhl instability threshold. The mode pair with the lowest threshold is called the critical one and usually only this pair goes unstable at the instability threshold.

In the following, we will introduce the relative of field amplitude $\varepsilon=h_{T} / h_{T}$ crit as the control parameter and consider only two parametric mode pairs. Mode 1 will be the critical one, and mode 2 with higher threshold field - the "weak" mode. Equations (3) may be then rewritten in the form

$$
\begin{aligned}
& \dot{a}_{0}=\left|\delta_{0}\right|\left|\delta_{1}\right| \varepsilon-\left(\eta_{0}+\mathrm{i} \Delta \omega_{0}\right) a_{0}-\mathrm{i} a_{1}^{2}-\mathrm{i}\left(\left|V_{0,2}\right| /\left|V_{0,1}\right|\right) a_{2}^{2}, \\
& \dot{a}_{1}=-\left(\eta_{1}+\mathrm{i} \Delta \omega_{1}\right) a_{1}-\mathrm{i} a_{1}^{*} a_{0}, \\
& \dot{a}_{2}=-\left(\eta_{2}+\mathrm{i} \Delta \omega_{2}\right) a_{2}-\mathrm{i}\left(\left|V_{0,2}\right| /\left|V_{0,1}\right|\right) a_{2}^{*} a_{0},
\end{aligned}
$$

where $a_{0, k}$ are proportional to $u_{0, k}$ and contain all constant phase factors, coming from the possibly complex values of the coupling coefficients. After dividing both sides of the equations by $\eta_{1}$ we work with the renormalized time $t^{\prime}=\eta_{1} t$.

\section{Results}

We solved Eqs. (5) numerically, employing a fourth-and-fifth order RungeKutta method with permanent step size and error control. Two sets of parameters, referred to as cases $A, B$, listed in Table, and various initial conditions were used. To characterize the chaotic solutions we calculated the Lyapunov exponents spectrum and the correlation dimension $D_{2}$ of the attractors. For the correlation dimension calculation, the attractors were first reconstructed from the time

TABLE

Numerical parameters for Eq. (5).

\begin{tabular}{l|c|c|c|c|c|c}
\hline \hline & $\eta_{0} / \eta_{1}$ & $\Delta \omega_{0} / \eta_{1}$ & $\Delta \omega_{1} / \eta_{1}$ & $\eta_{2} / \eta_{1}$ & $\Delta \omega_{2} / \eta_{1}$ & $\left|V_{0,2}\right| /\left|V_{0,1}\right|$ \\
\hline Case $A$ & 1.25 & -1.5 & 3.0 & 0.8 & 2.62 & 0.754 \\
Case $B$ & 1.25 & -1.5 & 2.8 & 0.8 & 2.9 & 0.496
\end{tabular}


dependence of $\left|a_{0}\right|$ according to the well-known Takens theorem [17] and then a modified Grassberger-Procaccia algorithm was used [18, 19]. The largest Lyapunov exponent $\lambda_{1}$ and the spectrum $\lambda_{1}>\lambda_{2}>\ldots>\lambda_{6}$ of Lyapunov exponents were calculated directly from the equations of motion, with the use of methods proposed in [20-22]. From the Lyapunov exponents spectrum we also calculated the Lyapunov or Kaplan-Yorke dimension

$$
D_{\mathrm{L}}=j+\frac{\sum_{i=1}^{j} \lambda_{i}}{\left|\lambda_{j+1}\right|},
$$

where $j$ is the greatest integer such that the sum in the numerator of the above equation remains positive. $D_{\mathrm{L}}$ always constitutes an upper bound for the correlation dimension value, and according to the well-known Kaplan-Yorke hypothesis, checked for simple chaotic systems, these two dimensions should be equal to each other [23].

For the two sets of parameters, cases $A$ and $B$ of Table, different behaviour of the chaotic solutions of Eqs. (5) with the increase in $\varepsilon$ was observed.

Case A. Auto-oscillations of the driven mode amplitude $\left|a_{0}\right|$ are observed for $\varepsilon>1.27$, and chaotic solutions for $\varepsilon>2.35$. Just above the threshold for auto-oscillations, only the critical mode amplitude oscillates and the "weak" mode amplitude decreases to zero independently on the choice of initial conditions, but for $1.34<\varepsilon<c a .2 .9$ amplitudes of all three modes are non-zero, and both the critical and the "weak" mode oscillate at the same level of excitation (Figs. 1a-d).

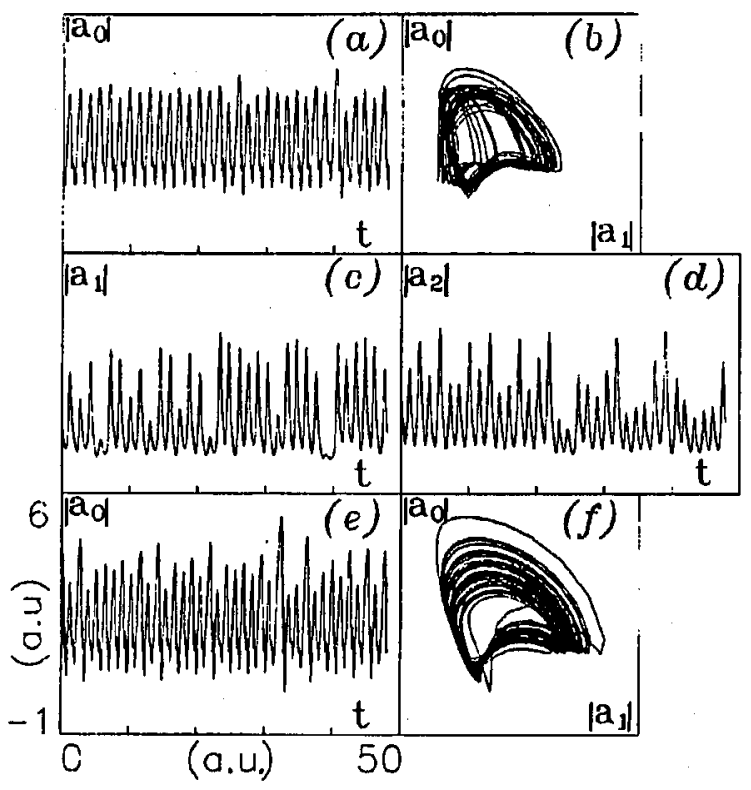

Fig. 1. Time series for mode amplitudes and chaotic attractors for case $A$ of Table. (a)-(d) for $\varepsilon=2.9$, (e)-(f) for $\varepsilon=3.2$. 
For $2.9<\varepsilon<3.0167$ the "weak" mode amplitude decreases from time to time to a very small value. The intervals of the small activity of the "weak" mode become longer with the rise of the rf field, and are separated by occasional bursts, when its amplitude is comparable with the critical mode amplitude. For $\varepsilon>3.0167$, the "weak" mode amplitude decreases to zero during the time evolution independently on the choice of initial conditions and the attractor is located at the hyperplane $\left|u_{2}\right|=\left|a_{2}\right|=0$. "Switching off" the "weak" mode changes the time series of the driven mode, which oscillates more irregularly (Fig. 1e); the chaotic attractor, projected on $\left|a_{0}\right|,\left|a_{1}\right|$ plane also changes its shape (Fig. 1f). For higher values of $\varepsilon$, in the neighbourhood of 4.2 and 4.7 , narrow periodic windows are observed. A complicated periodic solution is, however, destroyed from time to time by sudden bursts of the mode 2 amplitude, which in this case does not decrease to zero, but remains very small.

Figure 2a depicts the dependence of the correlation dimension of the attractor on the control parameter. For $2.3<\varepsilon<2.95$ the correlation dimension is contained between 2.6 and 3.1. For $\varepsilon>3.0$ it suddenly drops to the value smaller than 2.3 and only for higher rf field amplitude rises a little. This means that there exists a close connection between the correlation dimension and the number of modes, necessary to describe the system dynamics, i.e. the number of degrees of freedom of the system.
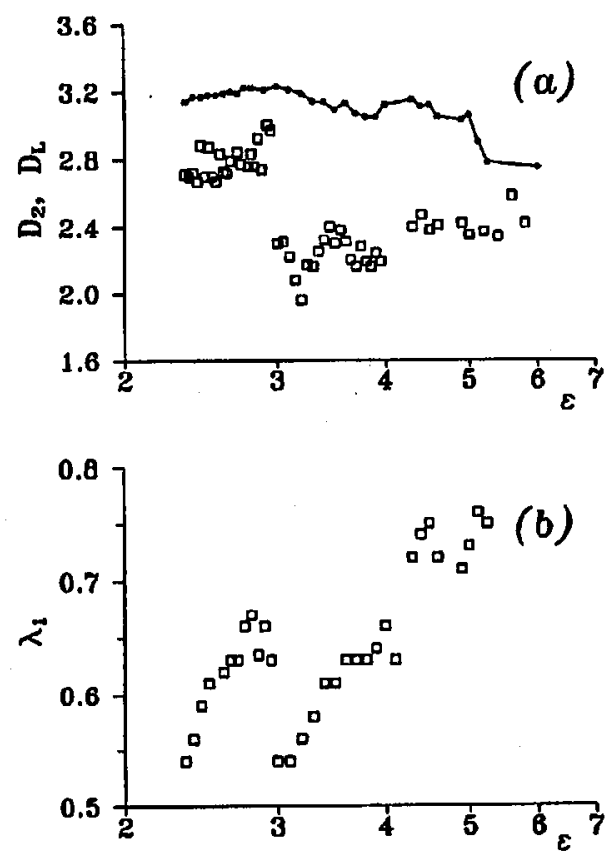

Fig. 2. (a) Correlation (squares) and Lyapunov (solid line) dimension of the chaotic attractor and (b) the largest Lyapunov exponent for case $A$ of Table. 
It is interesting to note that the Lyapunov dimension, calculated from Eq. (6), does not exhibit such sudden changes. For $2.3<\varepsilon<2.95$ its value $c a .3 .2$ is close to the correlation dimension value, in accordance with the Kaplan-Yorke hypothesis, but for $\varepsilon>3.0$ it decreases rather slowly, remaining considerably greater than the correlation dimension value (Fig. 2a). It is connected with the dependence of the Lyapunov spectrum on the control parameter.

For $2.3<\varepsilon<2.95$ the largest Lyapunov exponent is positive and increases with the rise of the rf field amplitude (Fig. $2 \mathrm{~b}$ ), and for $\varepsilon \approx 3.0$ it undergoes a sudden drop from the value ca. 0.66 to $c a .0 .54$. Positive value of this exponent indicates chaos. In all cases, the second largest Lyapunov exponent $\lambda_{2}$ is zero, as expected in continuous-time chaotic systems, which are not hyperchaotic. The decisive factor in the Lyapunov dimension evaluation is then the value of the third Lyapunov exponent $\lambda_{3}$. It turns out that this exponent for $2.3<\varepsilon<2.95$ remains negative and small; its absolute value is always smaller than $\lambda_{1}$ by an order of magnitude, yielding the $D_{\mathrm{L}}$ value greater than 3 , because three remaining Lyapunov exponents are big and negative.

For $\varepsilon>3.0$ the third Lyapunov exponent decreases very slowly with the rise of the control parameter, and this is why the Lyapunov dimension remains almost unaffected by the $\lambda_{1}$ drop. When we calculated the Lyapunov exponents spectrum for the system consisting only of two modes, the externally driven one and the critical one (four complex equations of motion) for $\varepsilon>3.0$, it turned out that the four Lyapunov exponents had values very close to $\lambda_{1}, \lambda_{2}, \lambda_{4}, \lambda_{6}$ of the original three-mode system (5), yielding the Lyapunov dimension between 2.2 and 2.3, again in accordance with the Kaplan-Yorke hypothesis. We then suppose that $\lambda_{3}, \lambda_{5}$ exponents of our system describe the contraction of the six-dimensional phase space in the directions perpendicular to the four-dimensional hyperplane, to which the motion of the system is constrained for $\varepsilon>3.0$. The part of the phase space which is accessible to the system has smaller dimensionality (four) than the full phase space (six) and in this reduced phase space the Kaplan-Yorke hypothesis is fulfilled. It should however be noted that in [8] the authors also observed a significant difference between the Lyapunov and correlation dimensions of their chaotic attractor (chaos in stroboscopic model for parallel pumping) and drew a conclusion that the Kaplan-Yorke hypothesis need not be valid in strongly chaotic systems.

Case $B$. Chaotic solutions are observed for $\varepsilon>2.1$, but for $\varepsilon<2.53$ only the critical mode amplitude is non-zero, and the attractor is located at the hyperplane $\left|u_{2}\right|=\left|a_{2}\right|=0$ (Fig. 3a,b). In the vicinity of $\varepsilon=2.53$ the "weak" mode occasionally bursts and, this time, the intervals between bursts shrink as the control parameter increases. For higher values of the rf field amplitude, independently on the choice of initial conditions, all modes have non-zero amplitudes (Fig. 3c-f), but two parametric modes behave in a different way than in case $A$. The critical mode is strongly excited and oscillates at the level comparable to the driven mode (Fig. 3e), while the "weak" mode only bursts from time to time (Fig. 3f), and these bursts, superimposed on the chaotic signal, produce a time series, which at first sight looks like a quasiperiodic one it is, however, chaotic, with positive largest Lyapunov exponent. Just above $\varepsilon=2.53$ these bursts are not so frequent and the "weak" 


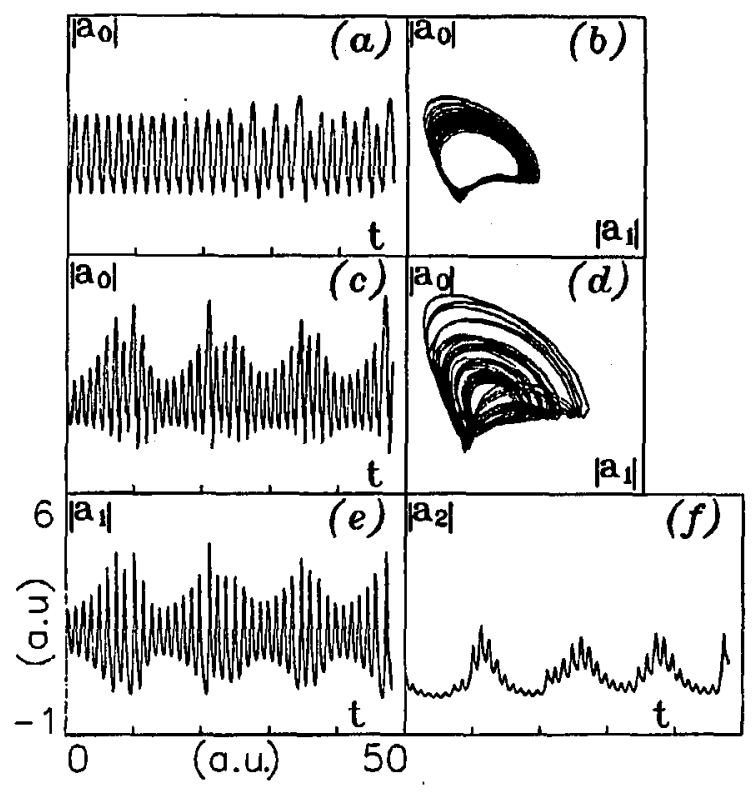

Fig. 3. Time series for mode amplitudes and chaotic attractors for case $B$ of Table. (a)-(b) for $\varepsilon=2.4$, (e)-(f) for $\varepsilon=3.1$.

mode amplitude oscillates chaotically at almost constant level, lower than the critical mode. In this range of $\varepsilon$ the correlation dimension of the attractor increases rapidly from $c a .2 .1$ to $c a .2 .8$.

For higher rf field amplitude the bursts become more frequent, and the correlation dimension decreases (Fig. 4a). The largest Lyapunov exponent behaves similarly (Fig. 4b). We found that if the critical rf field amplitude for the excitation of the "weak" mode was above the threshold for chaos, the decrease in the correlation dimension and largest Lyapunov exponent as well as appearance of bursts for increasing $\varepsilon$ followed independently on the particular choice of parameters in Eq. (5). The Lyapunov dimension, as in case $A$, does not show any sudden changes neither in the vicinity of $\varepsilon=2.53$ nor when the correlation dimension decreases (Fig. 4a). The reason is again the $\lambda_{2}, \lambda_{3}$ dependence on the control parameter, because $\lambda_{4} \ldots \lambda_{6}$ are big and negative. For $\varepsilon \leq 2.53$ the second exponent is zero and the third one slowly rises up to zero. For $\varepsilon>2.53$ both exponents are close to zero, and the second one for $\varepsilon>3.0$ assumes small positive values, an order of magnitude smaller than $\lambda_{1}$, suggesting either hidden quasiperiodicity (one positive, two zero exponents) or weak hyperchaos (two positive, one zero exponent). This produces $D_{\mathrm{L}}$ almost constant, between 3.0 and 3.2 over a wide range of the control parameter, again calling the Kaplan-Yorke hypothesis into question.

On-off intermittency. It is interesting to see what happens to the "weak" mode close to the critical value of the control parameter for its decay (case $A$, $\varepsilon_{\mathrm{c}} \approx 3.167$ ) or excitation (case $B, \varepsilon_{\mathrm{c}} \approx 2.53$ ). We will analyze this problem for case $A$. The time evolution of $\left|a_{2}\right|$ for a very long stretch of time is depicted for 

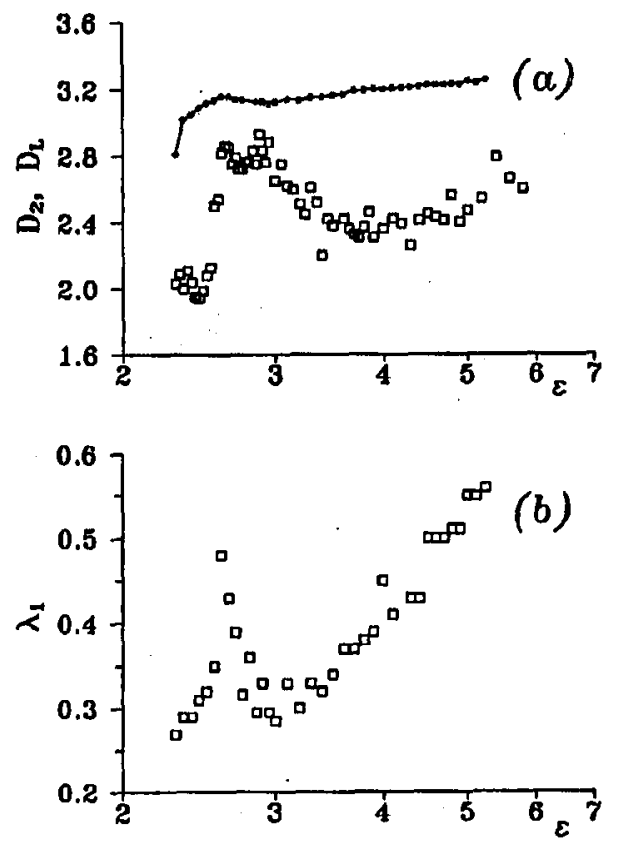

Fig. 4. (a) Correlation (squares) and Lyapunov (solid line) dimension of the chaotic attractor and (b) the largest Lyapunov exponent for case $B$ of Table.

$\varepsilon=2.99$ in Fig. $5 \mathrm{a}$ and 3.01 in Fig. $5 \mathrm{~b}$. In this very large time scale, in Fig. $5 \mathrm{a}$ the "weak" mode is strongly excited all the time, while in Fig. 5b periods of bursts and laminar, "flat" phases, when its amplitude remains practically equal to zero, can easily be distinguished. As already mentioned, when the control parameter still increases, approaching the critical value, periods of small activity of the "weak" mode grow longer and the bursts become rare. Dependence of the mean length of the laminar phase $\langle\tau\rangle$ on the difference $\left|\varepsilon-\varepsilon_{c}\right|$ is shown on the log-log plot of Fig. 6a (for $\varepsilon_{c}=3.0167$ ). Every point was obtained as an average over 20000 laminar phases from the solutions with 10 different, randomly chosen sets of initial conditions. It is evident that the points in Fig. 5a may be approximated by a straight line, suggesting that $\langle\tau\rangle \sim\left|\varepsilon-\varepsilon_{\mathrm{c}}\right|^{\gamma}$ with $\gamma=-1.05 \pm 0.2$. This value of $\gamma$ was obtained from the least squares method, but its error amounting to $20 \%$ was estimated after taking into consideration the uncertainty of $\varepsilon_{\mathrm{c}}$ evaluation. From our simulations it results that $\varepsilon_{c}>3.016$ and it should be smaller than 3.018 ; for this latter value $\left|a_{2}\right|$ always decreases to numerical zero, even after adding a small numerical noise to the solution (on the order of $10^{-300}$ ). More exact estimation of $\varepsilon_{\mathrm{c}}$ turns out to be difficult. As the difference between the actual and critical values of the rf field amplitude diminishes, the numerical calculations necessary for the proper evaluation of $\langle\tau\rangle$ become extremely time-consuming and we found them impossible to continue for $\left|\varepsilon-\varepsilon_{c}\right| \leq 5 \times 10^{-4}$. One should remember that usually such calculations are performed for low-dimensional maps, while here a system 

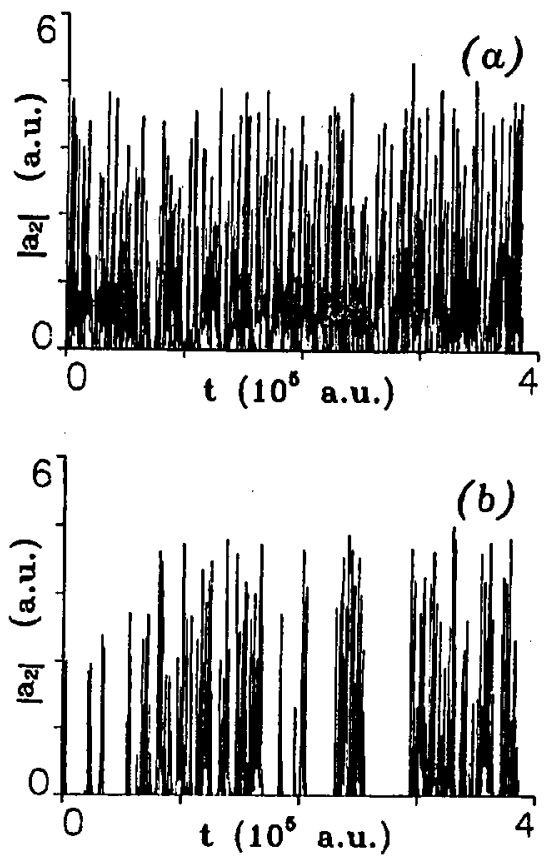

Fig. 5. Long-time evolution of the "weak" mode amplitude, case $A$ of Table, for (a) $\varepsilon=2.99$ and (b) $\varepsilon=3.01$.

of differential equations is solved, which substantially lengthens the computation time.

In Fig. $6 \mathrm{~b}$ the plot of $\ln N(\tau)$ vs. $\ln \tau$ is shown, where $N(\tau)$ is the number of laminar phases of length $\tau$. The total number of laminar phases was 75000 for $\varepsilon=3.01$. The dashed line corresponds to $N(\tau) \sim \tau^{-3 / 2}$. Both Figs. $6 \mathrm{a}$,b show that the transition between chaotic states with one or two parametric mode pairs excited results here from the so-called on-off intermittency $[12,13]$.

To understand this we should remind that such a phenomenon appears in chaotic and stochastically driven systems which possess quasi-invariant sets within their phase space, i.e. states near to which the system tends to spend long times [12]. Switching between bursts and extended periods of stasis is characteristic of the on-off intermittency. In our case, for Eqs. (5) we found that if initial conditions were chosen so that $a_{2} \equiv 0$, the system would evolve only on a four-dimensional hypersurface $a_{2}=a_{2}^{*}=0$, which is then an invariant set for the time evolution operator. It seems that for $\varepsilon>3.0167$ this invariant manifold is stable, but for $\varepsilon<3.0167$ it goes unstable which allows the appearance of bursts of the "weak" mode amplitude; coupling between the "weak" mode and the driven one is realized by the term $\left(\left|V_{0,2}\right| /\left|V_{0,1}\right|\right) a_{2}^{*} a_{0}$ in Eq. (5). An interesting feature of our case is that, contrary to most known examples, the invariant set is unstable below some critical value of the control parameter, and stable above this critical value, so laminar phases appear for decreasing, not increasing control parameter. Moreover, it was 

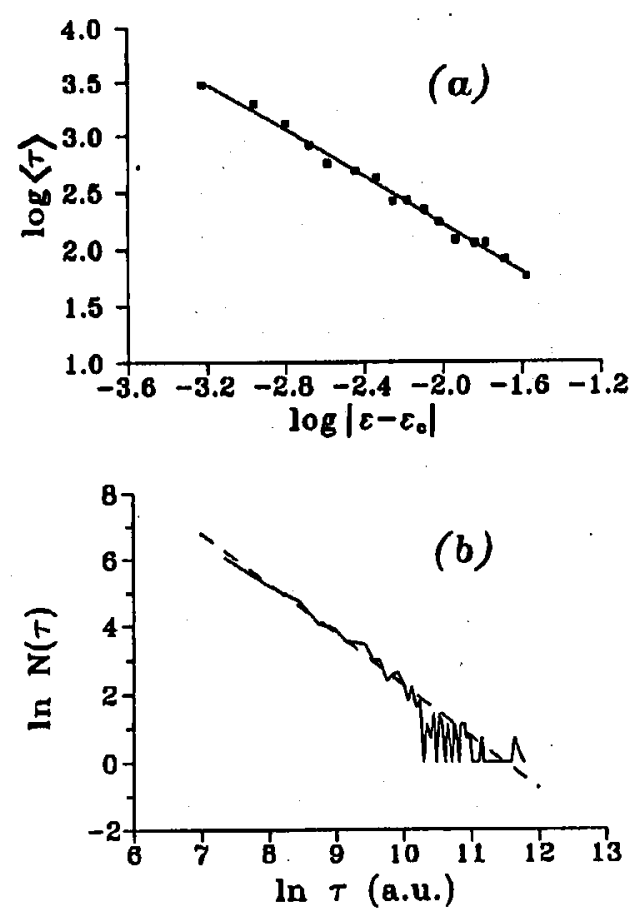

Fig. 6. Case $A$ of Table: (a) log-log plot of the mean laminar ("flat") phase length $\langle\tau\rangle$ vs. difference between $\varepsilon$ and the critical value of the control parameter for the "weak" mode extinction $\varepsilon_{\mathrm{c}}=3.0167$; the straight line is the least squares fit for $\log \langle\tau\rangle=$ $-1.05 \log \left|\varepsilon-\varepsilon_{c}\right|+0.11$. (b) ln-ln plot of the number $N(\tau)$ of laminar phases of length $\tau$ for $\varepsilon=3.01$; the dashed line results from $N(\tau)=A \tau^{-3 / 2}$ dependence with $\ln A=17.38$.

shown on the basis of chaotic and stochastic maps investigation [13] that the mean laminar phase length as a function of the control parameter should obey a power law with a critical exponent $\gamma=-1,\langle\tau\rangle=\alpha\left|\varepsilon-\varepsilon_{\mathrm{c}}\right|^{-1}+\delta$, where $\alpha, \delta$ are constants. Our value of $\gamma=1.05 \pm 0.2$ agrees well with the theory and the additive constant $\delta$, if any, is probably very small. It was also predicted in Ref. [13] that the distribution of laminar phases, i.e. the appearance probability for the laminar phase of a given length, should obey a power law $P(\tau) \sim N(\tau) \sim \tau^{-3 / 2}$ which agrees with our result, too.

\section{Discussion and conclusions}

In the preceding section we have shown that within a very simple model of chaos above the first-order Suhl instability threshold in ferromagnetic resonance it is possible to obtain non-trivial effects, which we now would like to compare to the experiments of Carroll et al. [7]. In these experiments, different volume and surface magnetostatic modes of an in-plane magnetized YIG (yttrium iron garnet) film, close in frequency to the uniform mode, were excited in resonance, and above their first-order Suhl instability threshold chaotic behaviour of the absorption in the sample was investigated. The most important result of our study is that we 
obtained sudden changes of the correlation dimension and maximum Lyapunov exponent with the rise of the relative rf field amplitude. The solutions in case $A$ resemble the dependence of these quantities on $\varepsilon$ for the $(3,1)$ surface mode instability and in case $B$ for the $(1,3)$ volume mode instability in the experiment [7].

Though the exact values of the correlation dimension and maximum Lyapunov exponents are not in perfect agreement with the experimental data, e.g. for the $(1,3)$ mode correlation dimension does not decrease for higher rf fields, the qualitative effect can be seen clearly. Similar effects, but with much higher correlation dimension, suggesting more interacting modes, were also observed in spherical samples, driven in coincidence regime, and a hypothesis was put forward that they are connected with the change of the number of excited parametric mode pairs [24]. In the analysis of their experimental results Carroll et al. [7] suggested that such sudden changes of the correlation dimension and Lyapunov exponent may require change of the coupling between the driven mode and the pairs of parametric modes, leading to the bifurcations in the system of interacting modes. Here we have shown that the interactions among modes need not change in order to produce such effects in the time series, and that it is possible to obtain them from a very simple model with properly chosen parameters. The nature of the transition between different chaotic states was not investigated in the experiment, but from the discussion in Sec. 3 it results that such transition may appear as a result of the on-off intermittency.

Similar effect of "switching on" additional modes with the rise of the rf field amplitude was also observed in numerical modelling of chaos in parallel pumping with the use of the stroboscopic model [8], but the reverse effect of "switching off" one mode, described in our paper as case $A$, was not reported. In that resonance configuration the rf field excites directly parametric modes with half the pumping frequency, which interact via the four-mode interactions, so the coupling mechanism is completely different. Excitation of an additional mode pair leads there to the appearance of hyperchaos (two Lyapunov exponents greater than zero). In our case $B$ such chaos-hyperchaos transition is rather not observed, because for $\varepsilon>3.0$ the second Lyapunov exponents, though remains positive, is very close to zero, while in Ref. [8] it was only by a factor two to three smaller than the largest one. Like in [8], in our case the Lyapunov dimension, which according to the Kaplan-Yorke hypothesis should be equal to the correlation dimension, turned out to be in general greater than the dimension calculated from the Grassberger-Procaccia algorithm and varied more smoothly with the rise of control parameter.

We end with some remarks concerning the validity of our model in describing chaos above the first-order Suhl instability threshold. This model yields exact results only at the instability threshold, because for higher rf field amplitude other three-mode and four-mode interactions should be taken into consideration. However, these interactions are weak in comparison with the basic ones and it should be possible to understand, at least qualitatively, the magnetic system behaviour above the threshold without including them in the model. Neglect of all additional. interactions considerably reduces the time of numerical calculations, too. Such simple model was already considered in the last paper of Ref. [7] in connection with the 
experimental results presented there, but the authors constrained themselves only to some general remarks. A more complicated model was proposed by Wiese and Benner [4]. In their model, developed to describe chaos in ferromagnetic spheres under coincidence regime, pairs of parametric modes strongly interact not only with the externally driven uniform mode, but also with a mode which is close in frequency to it. In the samples of other shapes the modes "almost degenerate" with the driven one may also exist, and such interactions are next source of nonlinearity, not explicitely included in our equations of motion.

Auto-oscillations and chaos in coincidence regime were observed experimentally and predicted from the model in Ref. [4] only if the frequency spacing among neighbouring parametric modes, resulting from finite sample dimensions, was comparable with the mode damping. It turned out that both in the model of Wiese and Benner and in our model it was necessary to include non-zero detuning from $\omega / 2$ for the parametric modes, as in Table, to obtain auto-oscillations and chaos. It is known that the spontaneous oscillations of the magnetization appear as a result of the Hopf bifurcation [2]. In our model, but with only one pair of parametric modes included, it was verified by performing a stability analysis for the fixed point of Eq. (5) above the instability threshold that this bifurcation cannot appear if all detunings are zero. Thus we include non-zero detunings for all modes, treating them as free and important parameters, resulting e.g. from the finiteness of the sample dimensions. It should be noted that Rezende et al. also include detunings for the parametric modes in their two-mode model of auto-oscillations and chaos in parallel pumping to explain the auto-oscillations frequency dependence on the sample dimensions [6], though in this resonance configuration their equations of motion for the mode amplitudes exhibit periodic and chaotic behaviour even with all detunings equal to zero.

Apart from this dependence, the auto-oscillations frequency is proportional to the parametric mode damping [2]. In Ref. [7], just above the Suhl threshold the experimental time series were periodic, with the frequency $1-100 \mathrm{kHz}$. In this paper, we obtained auto-oscillations e.g. in case $A$ for $\varepsilon>1.27$, which may be connected with the assumed simplicity of our model. If in Eq. (5) the value of $\eta_{1}=1.0 \times 10^{6} \mathrm{~s}^{-1}$ was assumed, which is a reasonable order of magnitude for this quantity, the obtained frequency would be $300-400 \mathrm{kHz}$, so it is necessary to decrease the mode damping at least 3-5 times; this problem is common for almost all simple numerical models of chaos in ferromagnetic resonance (the exception is [9]) and remains unsolved in our model, too.

To conclude, in this paper we have shown that using a very simple model of chaos above the first-order Suhl instability threshold, containing only three-mode resonant interactions, it is possible to model non-trivial effects of sudden changes of the correlation dimension and largest Lyapunov exponent. They are connected with the change of the phase space part occupied by the chaotic attractor, which may result from the on-off intermittency, leading to the excitation or decay of certain parametric mode pairs. The results are in qualitative agreement with the experimental investigation of chaos in ferromagnetic resonance in thin magnetic films. 


\section{Acknowledgment}

One of us (A.K.) was supported by the State Committee for Scientific Research (Republic of Poland), grant No. 2 P302 042 06. The authors thank Dr. hab. J.J. Żebrowski for providing a FORTRAN code for the correlation dimension calculation and helpful discussions.

\section{References}

[1] G. Gibson, C. Jeffries, Phys. Rev. A 29, 811 (1984).

[2] X.Y. Zhang, H. Suhl, Phys. Rev. B 38, 4893 (1987).

[3] P.H. Bryant, C.D. Jeffries, K. Nakamura, Phys. Rev. A 38, 4223 (1988).

[4] G. Wiese, H. Benner, Z. Phys. B, Condens. Matter 79, 119 (1990).

[5] A. Azevedo, S.M. Rezende, Phys. Rev. Lett. 66, 1342 (1991).

[6] S.M. Rezende, F.M. de Aguiar, A. Azevedo, J. Appl. Phys. 73, 6805 (1993).

[7] T.L. Carroll, L.M. Peccora, F.J. Rachford, J. Appl. Phys. 67, 5630 (1990); J. Appl. Phys. 69, 5727 (1991); J. Appl. Phys. 70, 3197 (1991).

[8] H.R. Moser, P.F. Meier, F. Waldner, Phys. Rev. B 47, 217 (1993).

[9] R.D. McMichael, P.E. Wigen, Phys. Rev. B 42, 6723 (1990).

[10] H. Suhl, J. Phys. Chem. Solids 1, 209 (1957).

[11] P. Kabos, G. Wiese, C.E. Patton, Phys. Rev. Lett. 72, 2093 (1994).

[12] N. Platt, E.A. Spiegel, C. Tresser, Phys. Rev: Lett. 70, 279 (1993).

[13] J.F. Heagy, N. Platt, S.M. Hammel, Phys. Rev. E 49, 1140 (1994).

[14] F. Waldner, J. Phys. C, Solid State Phys. 21, 1243 (1988).

[15] V. Zakharov, V. Lvov, S.S. Starobinets, Usp. Fiz. Nauk 114, 609 (1974) [Sov. Phys.-Usp. 17, 896 (1975)].

[16] A. Krawiecki, A. Sukiennicki, Acta Phys. Pol. A 83, 505 (1993).

[17] F. Takens, in: Dynamical Systems and Turbulence, Lecture Notes in Mathematics, Vol. 898, Eds. D.A. Rand, L.S. Young, Springer, New York 1981, p. 366.

[18] P. Grassberger, I. Procaccia, Phys. Rev. Lett. 50, 346 (1983).

[19] J. Theiler, Phys. Rev. A 34, 2427 (1986).

[20] G. Benettin, L. Galgani, J.M. Strelcyn, Phys. Rev. A 14, 2338 (1976).

[21] G. Benettin, L. Galgani, A. Giorgilli, J.M. Strelcyn, Meccanica 15, 9, 21 (1980).

[22] A. Wolf, J.B. Swift, H.L. Swinney, J.A. Vastano, Physica D 16, 285 (1985).

[23] J. Kaplan, J. Yorke, in: Functional Differential Equations and Approximation of Fixed Points, Lecture Notes in Mathematics No. 730, Eds. H.O. Peitgen, H.O. Walther, Springer, New York 1979, p. 204.

[24] H. Benner, unpublished lecture at VI Symp. on Statistical Physics, Zakopane (Poland) 1993. 